\title{
Utilização de Modelos Matemáticos para Estimar a Retenção de Minerais em Cabras durante a Gestação 1
}

\section{Roberto Germano Costa ${ }^{2}$, Kleber Tomás de Resende ${ }^{3}$, Marcelo Teixeira Rodrigues ${ }^{4}$, Cláudio José Borela Espechit ${ }^{4}$, Augusto César de Queiroz ${ }^{4}$}

\begin{abstract}
RESUMO - O trabalho foi realizado com o objetivo de estimar a retenção de elementos minerais durante a gestação de cabras com um ou dois fetos, utilizando-se diferentes modelos matemáticos. A estimativa de retenção foi baseada na diferença entre o total de cada mineral depositado no feto, útero, membranas, fluídos fetais e glândula mamária dos animais nas diferentes etapas da gestação e o total de cada mineral armazenado nas cabras vazias, utilizando-se os modelos de predição: $\ln \mathrm{Y}=\mathrm{A}-\mathrm{B} \mathrm{e}^{-\mathrm{C} \mathrm{X}} ; \mathrm{Y}=\mathrm{A} \mathrm{e}^{\mathrm{B} X}$ e ln $\mathrm{Y}=\mathrm{A}+\mathrm{Bx}$ $+\mathrm{Cx}^{2}$, em que $\mathrm{x}=$ tempo de gestação. Comparando-se a estimativa com os valores reais obtidos, verificou-se que o modelo proposto $\ln =\mathrm{A}+\mathrm{Bx}+\mathrm{Cx}^{2}$, explicou com coerência e precisão o comportamento biológico da retenção de minerais durante todas as fases da gestação.
\end{abstract}

Palavras-chave: cabras, gestação, minerais, retenção de minerais

\section{Utilization of Mathematic Models to Estimate the Minerals Retention during the Pregnancy of Goats}

\begin{abstract}
This work was carried out with the purpose to evaluate the minerals retention during the pregnancy of goats with one or two foetus. The estimate of retention was based in the difference between the total of each mineral stored in the foetus, uterus, membranes, fetals fluids and mammary gland of animals in the differents stages of pregnancy and the total of each mineral stored in the empty goats, using the model of prediction: $\ln \mathrm{Y}=\mathrm{A}-\mathrm{B} \mathrm{e}^{-\mathrm{C} X} ; \mathrm{Y}=\mathrm{A} \mathrm{e}^{\mathrm{B} X}$ and $\ln \mathrm{Y}=\mathrm{A}+\mathrm{Bx}+\mathrm{Cx} 2$, where $\mathrm{x}=$ time of pregnancy. The comparison of the estimative with the real values obtained show that the suggest model explained with coherence and precision the biological behavior of minerals retention during all pregnancy.
\end{abstract}

Key Words: goats, pregnancy, minerals, minerals retention

\section{Introdução}

Os minerais representam um componente essencial para a dieta dos caprinos e influenciam de modo marcante a sua produtividade, podendo em alguns casos ou regiões com solos deficientes, ser limitantes econômica e biologicamente e afetar inclusive a sobrevivência do animal. Durante a gestação, o nível nutricional tem extrema importância, sobretudo nos últimos 45 dias, quando os tecidos fetais têm maior desenvolvimento (Kadu \& Kaikini, 1987). Se os nutrientes não forem suficientes nas últimas semanas que antecedem o parto, a concorrência entre mãe e feto poderá levar ao aborto.

A gestação é uma fase importante na vida das fêmeas, já que as transformações que ocorrem afetam não somente o aparelho reprodutivo, mas todo o organismo animal. O metabolismo da mãe sofre profun- das modificações, pois deve prover o feto de todos os princípios nutritivos em quantidades suficientes, embora haja, durante a gestação, uma notável melhoria nos processos de absorção do conduto alimentício, particularmente na assimilação de substâncias minerais.

O ARC (1980) recomenda que para se calcular as quantidades de nutrientes depositadas no útero grávido, em sucessivos estádios da gestação, faz-se necessário conhecer: a quantidade total de nutrientes no feto; a quantidade adicional depositada nas membranas fetais, fluidos e parede uterina e a quantidade de nutrientes depositada em diferentes estádios da gestação.

Estudos sobre a composição química de vários componentes, em diferentes estádios da gestação, foram desenvolvidos por Graham (1964), Lodge \& Heaney (1973), Rattray et al. (1974) e Robinson et al. (1977), em ovinos. Entretanto, na literatura disponí-

\footnotetext{
1 Parte da tese apresentada pelo primeiro autor ao Doutorado em Zootecnia da FCAV-UNESP, Campus de Jaboticabal-SP

2 Departamento de Agropecuária do Campus IV da UFPB, Bananeiras-PB, CEP 58.220-000. E.mail: rgermano@cft.ufpb.br

3 Departamento de Zootecnia da FCAV/UNESP, Campus de Jaboticabal-SP, CEP 14.870-000.

4 Departamento de Zootecnia da UFV, Viçosa-MG. CEP: 36.570-000
} 
vel, pouco se encontra a respeito das quantidades de nutrientes retidos no útero grávido de cabras. Alguns estudos dessa natureza foram desenvolvidos por Anderson et al. (1981), Kadu \& Kaikini(1987), Fandino (1989) e Moulin (1991), sem abordarem, contudo, aspectos relacionados às exigências de minerais.

Diante do exposto, justifica-se a realização de pesquisas sobre nutrientes inorgânicos em caprinos, principalmente na fase reprodutiva, considerando-se a importância dos elementos minerais na gestação dos animais domésticos. Portanto, objetivou-se neste trabalho avaliar a retenção de minerais para cabras em diferentes estádios de gestação com um e dois fetos, utilizando-se diferentes modelos matemáticos para estimativa desses minerais.

\section{Material e Métodos}

O trabalho foi desenvolvido por intermédio de uma parceria entre a Universidade Estadual Paulista - UNESP, Campus de Jaboticabal-SP e a Universidade Federal de Viçosa-UFV. O experimento foi conduzido com um grupo de 120 fêmeas, com pesomédio de $42 \mathrm{~kg}$, não gestantes, sem raça definida (SRD). Após quatro semanas de adaptação às instalações e a um mesmo tipo de ração, cinco cabras foram submetidas ao abate, para efeito de análise comparativa das carcaças. As demais fêmeas foram submetidas à sincronização de cio e quarenta e cinco dias após a primeira cobrição foi realizado o diagnóstico da gestação e a contagem do número de fetos, por meio de laparotomia.

Comprovada a gestação e determinado o número de embriões de cada fêmea, foram constituídos 10 lotes de 5 animais cada um, totalizando 50 animais, descartando-se as cabras excedentes. Metade dos lotes era formada de gestações simples e a outra metade de gestações de dois fetos.

Aos 50 dias, dois dos 10 lotes, com número diferente de fetos, foram tomados ao acaso e abatidos. A partir desse momento, 4 grupos de 5 cabras gestantes de um ou dois fetos continuaram com a alimentação ad libitume foram destinados ao abate aos 100 e 140 dias. Os 4 grupos restantes, também de um ou dois fetos e abates previstos para 100 e 140 dias, passaram a receber alimentação restrita, na base de $20 \%$ acima da exigência de energia metabolizável para mantença. A partir dos 50 dias de gestação até o abate, as cabras foram mantidas em gaiolas individuais, utilizando-se em todos os trata- mentos a mesma ração, contendo $2531 \mathrm{kcal}$ de EM/ $\mathrm{kg}$ de MS e $13 \%$ de proteína bruta na MS, cuja quantidade era ajustada semanalmente, de acordo com o aumento de peso dos animais.

A ração continha 2,36\% de uma mistura mineral, que possuía a seguinte composição percentual: sal comum-37,3; fosfato bicálcico- 46,95; sulfato de magnésio- 13,91 ; sulfato de zinco- 0,86 ; sulfato de magnésio- 0,747 , sulfato de cobre- 0,19 ; calcário0,03 ; sulfato de cobalto- 0,0068 ; iodato de potássio0,00582; selenito de sódio- 0,00106.

Após o sacrifício dos animais, foram removidos a glândula mamária e o útero. $\mathrm{O}$ trato reprodutivo foi separado na cérvice e dissecado em útero, feto, membranas e fluídos fetais, pesados separadamente. O conteúdo gastrintestinal foi eliminado, para determinação do peso do corpo vazio e, em seguida, o aparelho digestivo juntamente com o sangue, vísceras, cabeça, patas, couro e carcaça foram congeladas, triturados e homogeneizados em "cutter" de $30 \mathrm{HP}$ e $1775 \mathrm{rpm}$ e amostrados.

$\mathrm{O}$ útero e as membranas fetais foram moídos juntos e misturados com o líquido placentário. Os fetos de 100 e 140 dias de gestação foram triturados separadamente em moedor de carne. Os fetos de 50 dias de concepção, em razão do pequeno peso individual, foram triturados em grupo (5 animais do mesmo tratamento). A glândula mamária foi moída com a pele e submetida ao mesmo procedimento anterior.

Após a secagem, esse material foi desengordurado em aparelho "Soxhlet" e triturado em moinho de bola. Em seguida, foi determinada a matéria seca de cada constituinte da gestação e preparada a solução mineral pela da técnica via úmida (Silva, 1998). A solução mineral foi encaminhada ao laboratório para determinação dos macroelementos: cálcio (ca), fósforo $(\mathrm{P})$, sódio $(\mathrm{Na})$, potássio $(\mathrm{K})$, magnésio $(\mathrm{Mg})$, enxofre $(\mathrm{S})$, e dos microelementos: ferro ( $\mathrm{Fe}$ ) e zinco ( $\mathrm{Zn}$ ). Os elementos $\mathrm{P}$ e $\mathrm{S}$ foram analisados por colorimetria, enquanto os demais minerais foram determinados por espectrofotometria de absorção atômica, conforme metodologia estabelecida pela AOAC (1975).

As estimativas de retenção foram baseadas na diferença entre o total de cada mineral depositado no feto, útero, membranas, fluídos fetais, glândula mamária e corpo vazio dos animais nas diferentes etapas da gestação, e o total de cada mineral armazenado nas cabras vazias (testemunha), conforme a metodologia estabelecida pelo ARC (1980).

O delineamento experimental utilizado foi um 
fatorial 3 × 2 × 2 , envolvendo os fatores: época de gestação (3); tipo de alimentação (2) e número de fetos (2). Os dados dos minerais retidos nos diferentes constituintes da gestação foram submetidos a uma análise de regressão, para se estabelecer equações de predição em função dos fatores estudados e, por conseguinte, a deposição diária de cada elemento mineral nesses constituintes. Para tanto, foram testados três diferentes modelos de predição:

$$
\begin{array}{ll}
\ln \mathrm{Y}=\mathrm{A}-\mathrm{B} \mathrm{e}^{-\mathrm{C} X} & \text { (Modelo 1) } \\
\mathrm{Y}=\mathrm{A} \mathrm{e}^{\mathrm{B} \mathrm{X}} & \text { (Modelo 2) } \\
\ln \mathrm{Y}=\mathrm{A}+\mathrm{BX}+\mathrm{Cx}^{2} & \text { (Modelo 3) }
\end{array}
$$

em que: $\mathrm{Y}=$ conteúdo de mineral no componente da gestação; A, B e C = constantes obtidas pela análise de regressão; $X=$ tempo de gestação.

Os parâmetros das curvas de retenção de minerais foram obtidos por intermédio de funções nãolineares, pelo método dos quadrados mínimos. $\mathrm{O}$ programa utilizado foi o CURVAS, uma versão em BASIC desenvolvida por Borato (1984) e adaptada pela FCAV-UNESP.

Por se tratar de um processo interativo e fortemente dependente dos valores iniciais, as equações foram linearizadas e por intermédio do procedimento REG do SAS (1989), calculou-se uma solução inicial para os modelos, dada como ponto de partida para as interações do programa CURVAS.

\section{Resultados e Discussão}

Os resultados das análises de regressão do conteúdo de cada mineral no útero grávido mais úbere, em função do tempo de gestação, são apresentados nas Tabelas 1 e 2 . Todos os parâmetros das equações, obtidos nos três modelos estudados, tanto para um feto quanto para dois fetos, foram altamente significativos $(\mathrm{P}<0,01)$.

O Modelo 1 vem sendo tradicionalmente utilizado para determinar a retenção de minerais em ovinos e bovinos (ARC, 1965, 1980; AFRC, 1991). Já o Modelo 2 é comumente utilizado para descrever a dinâmica do desenvolvimento fetal em ovinos (Koong et al., 1975).

O Modelo 3 foi proposto partindo-se da premissa que a retenção de minerais (Y) apresenta uma taxa de crescimento variável. Essa taxa inicialmente é baixa, aumenta até um determinado ponto e depois começa a decrescer, o que seria conveniente a utilização de um modelo matemático para "Y" que tivesse um ponto de inflexão nessa região. Ademais, dependendo do mineral estudado, essa taxa pode até mesmo tornar-se negativa, como é o caso dos minerais sódio e potássio (Ferrel et al., 1982).

Como a quantidade retida de determinado mineral, durante a gestação, é sempre positiva, o uso de um modelo exponencial representaria esse compor-

Tabela 1 - Parâmetros e coeficientes de determinação das equações de regressão obtidas para estimar a retenção de minerais no útero grávido mais úbere, em função do tempo de gestação de cabras com um feto, alimentadas ad libitum

\begin{tabular}{|c|c|c|c|c|c|c|c|c|c|}
\hline $\begin{array}{l}\text { Modelo } \\
\text { Model }\end{array}$ & $\begin{array}{l}\text { Parâmetro } \\
\text { Parameter }\end{array}$ & $\mathrm{Ca}(\mathrm{g})$ & $P(g)$ & $\mathrm{Na}(\mathrm{g})$ & K (g) & $\mathrm{Mg}(\mathrm{g})$ & S (g) & $\mathrm{Fe}(\mathrm{mg})$ & $\mathrm{Zn}(\mathrm{mg})$ \\
\hline Modelo 1 & A & $-7,82410$ & $-8,23847$ & 3,51054 & 4,48937 & 2,78971 & 3,88017 & $-4,37053$ & $-5,54291$ \\
\hline Model 1 & B & $-6,53500$ & $-7,05112$ & 6,94601 & 7,05287 & 7,25550 & 7,17565 & $-6,36966$ & $-6,54171$ \\
\hline $\ln Y=A-B e^{-C} x$ & $\mathrm{C}$ & $-0,00403$ & $-0,00325$ & 0,01436 & 0,00930 & 0,00885 & 0,00844 & $-0,00372$ & $-0,00322$ \\
\hline $\mathrm{R}^{2}$ & 0,98332 & 0,93825 & 0,93325 & 0,92765 & 0,87124 & 0,90828 & 0,85128 & 0,87869 & \\
\hline Modelo 2 & A & & 0,16829 & & & & & & 1,60860 \\
\hline Model 2 & B & 0,04213 & 0,03332 & 0,02125 & 0,02667 & 0,03463 & 0,02654 & 0,03661 & 0,03036 \\
\hline$Y=A e^{B} x$ & $\mathrm{R}^{2}$ & 0,98398 & 0,93906 & 0,92531 & 0,92742 & 0,90119 & 0,91560 & 0,85126 & 0,87971 \\
\hline Mode & A & -6 & & & & & & & $-1,33301$ \\
\hline Model 3 & B & 0,11152 & 0,06649 & 0,06639 & 0,07706 & 0,06678 & 0,01291 & 0,02256 & 0,06294 \\
\hline $\ln Y=A+B x+C x^{2}$ & $\mathrm{C}$ & $-0,00029$ & $-0,00014$ & $-0,00020$ & $-0,00022$ & $-0,00014$ & 0,00006 & 0,00006 & $-0,00014$ \\
\hline $\mathrm{R}^{2}$ & 0,98453 & 0,94000 & 0,93429 & 0,93230 & 0,90170 & 0,91635 & 0,85134 & 0,88056 & \\
\hline
\end{tabular}

Table 1 - Parameters and coefficients of determination of the obtained regression equation to estimate the minerals retention in the pregnant uterus plus ubber, in function of gestation time of goats with one foetus, fed ad libitum

R. Bras. Zootec., v.32, n.2, p.425-430, 2003 


\begin{tabular}{|c|c|c|c|c|c|c|c|c|c|}
\hline $\begin{array}{l}\text { Modelo } \\
\text { Model }\end{array}$ & $\begin{array}{l}\text { Parâmetro } \\
\text { Parameter }\end{array}$ & $\mathrm{Ca}(\mathrm{g})$ & $P(g)$ & $\mathrm{Na}(\mathrm{g})$ & $\mathrm{K}(\mathrm{g})$ & $\mathrm{Mg}(\mathrm{g})$ & $S(g)$ & $\mathrm{Fe}(\mathrm{mg})$ & $\mathrm{Zn}$ (mg) \\
\hline \multirow{4}{*}{$\begin{array}{l}\text { Modelo } 1 \\
\text { Model } 1 \\
\ln \mathrm{Y}=\mathrm{A}-\mathrm{Be}^{-\mathrm{C}} \mathrm{x}\end{array}$} & A & $-4,29169$ & $-7,61290$ & $-5,66230$ & $-7,40986$ & 2,84533 & $-8,56979$ & $-3,68301$ & $-5,36421$ \\
\hline & $\mathrm{B}$ & $-3,31615$ & $-6,89901$ & $-6,10271$ & $-7,08501$ & 5,74559 & $-7,07385$ & $-5,61980$ & 6,86286 \\
\hline & $\mathrm{C}$ & $-0,00686$ & $-0,00332$ & $-0,00257$ & $-0,00279$ & 0,00870 & $-0,00302$ & $-0,00451$ & $-0,00302$ \\
\hline & $\mathrm{R}^{2}$ & 0,88105 & 0,92658 & 0,89592 & 0,94657 & 0,75598 & 0,94781 & 0,93800 & 0,87784 \\
\hline \multirow{3}{*}{$\begin{array}{l}\text { Modelo } 2 \\
\text { Model } 2 \\
\mathrm{Y}=\mathrm{Ae}^{\mathrm{B}} \mathrm{x}\end{array}$} & A & 0,0588 & 0,2601 & 1,1678 & 0,4762 & 0,0188 & 0,1342 & 2,2927 & 2,7440 \\
\hline & B & 0,0514 & 0,0337 & 0,0209 & 0,0272 & 0,0377 & 0,0303 & 0,0433 & 0,0293 \\
\hline & $\mathrm{R}^{2}$ & 0,8813 & 0,9281 & 0,9040 & 0,9501 & 0,8777 & 0,9498 & 0,9383 & 0,8805 \\
\hline \multirow{4}{*}{$\begin{array}{l}\text { Modelo } 3 \\
\text { Model } 3 \\
\ln \mathrm{Y}=\mathrm{A}+\mathrm{Bx}+\mathrm{Cx}^{2}\end{array}$} & A & $-4,42149$ & $-4,18087$ & $-3,43583$ & $-4,14669$ & $-6,18928$ & $-4,22510$ & $-0,04511$ & $-2,44041$ \\
\hline & B & 0,07883 & 0,08362 & 0,08757 & 0,08829 & 0,07639 & 0,06979 & 0,05848 & 0,09069 \\
\hline & $\mathrm{C}$ & $-0,00012$ & $-0,00021$ & $-0,00029$ & $-0,00026$ & $-0,00016$ & $-0,00017$ & $-0,00006$ & $-0,00026$ \\
\hline & $\mathrm{R}^{2}$ & 0,88139 & 0,92969 & 0,93518 & 0,95794 & 0,87830 & 0,95212 & 0,93834 & 0,88524 \\
\hline
\end{tabular}

tamento. Além disso, um polinômio de $2^{\underline{Q}}$ grau daria condições ao modelo de se adaptar àquelas situações em que a taxa de retenção se torna negativa.

O modelo proposto apresenta tais características, e essas foram as razões para a sua proposição, sem desconsiderar, evidentemente, a existência de outros modelos que apresentam características iguais ou semelhantes e que também poderiam ser adotados.

Os três modelos propostos apresentaram elevados coeficientes de determinação $\left(\mathrm{R}^{2}\right)$, a maioria deles ostentando valores acima de $90 \%$. Merece consideração o fato de que em cada elemento mineral, isoladamente, esses coeficientes apresentaram o mesmo comportamento, embora o modelo 3 tenha demonstrado uma ligeira superioridade em relação aos demais.

Observando-se os dados referentes ao número de fetos, independentemente do modelo utilizado, verificou-se que esse comportamento também foi semelhante, à exceção do cálcio, que nas gestações com dois fetos, os modelos apresentaram coeficientes inferiores. Vale ressaltar, porém, que a utilização do coeficiente de determinação para escolha de curvas de ajuste nem sempre é uma boa medida. Doti \& Adibi (1988) afirmaram que freqüentemente é necessário, na estimativa de equações de regressão, selecioná-las considerando uma variedade de alternativas de aproximações estatísticas. Infelizmente, o exame do valor $\mathrm{R}^{2}$, entre as várias alternativas, traz pequena orientação nessa seleção (Willet \& Singer,
1988). Os valores $R^{2}$ não são geralmente aplicados quando eles são usados para comparar o poder explanatório de equações que têm diferentes formas funcionais (Anderson-Sprecher, 1994).

Nas Tabelas 3 e 4 são feitas comparações entre a retenção de minerais observada no útero grávido mais úbere e as suas correspondentes estimativas, obtidas pelas equações propostas para um e dois fetos, respectivamente. A análise dos dados dessas tabelas já sugere, portanto, uma diferenciação entre os modelos propostos.

Aos 50 dias de gestação, os Modelos 1 e 2 superestimaram a retenção dos minerais, indistintamente, enquanto as estimativas do Modelo 3 foram as que mais se aproximaram dos valores observados no experimento, sobretudo nas gestações com dois fetos. Todavia, como o primeiro terço da gestação é a fase em que, quantitativamente, a retenção de minerais é mínima, a utilização de qualquer uma das estimativas não provocaria tantos prejuízos, uma vez que os animais estariam recebendo suprimento de minerais acima das suas exigências, porém em quantidades pouco expressivas. Field \& Suttle (1967) afirmam que a taxa de deposição de minerais no início da gestação é pequena e, embora erros relativamente grandes sejam cometidos, nessa fase, seus efeitos na exigência total de minerais pouco significam.

Aos 100 dias de gestação, o comportamento inverteu. Os Modelos 1 e 2 subestimaram a retenção de minerais. No entanto, o Modelo 3 permaneceu apre- 
sentando melhores estimativas e, na maioria dos elementos, os valores estimados coincidiram com os observados. Em contrapartida, aos 140 dias de gestação, os 3 modelos apresentaram comportamento semelhante, com estimativas precisas dos elementos retidos no útero grávido mais úbere.

Analisando-se o comportamento global da retenção de elementos minerais, estimado pelos três modelos propostos, em todas as fases de gestação estudadas e, comparando-se com os valores reais obtidos no experimento, verificou-se que o Modelo 3 explicou com maior coerência e precisão o comportamento biológico da retenção de elementos minerais durante toda a gestação, critério esse que deve ser adotado na escolha de uma equação de regressão (Doti \& Adibi, 1988; Willet\& Singer, 1988; AndersonSrecher, 1994).

Merece destaque o fato de que, dos 100 aos 140 dias de gestação, a deposição de minerais para gestações com um e dois fetos representou, respectivamente: 80,9 e 86,9\% (Ca); 72,6 e 73,0\% (P); 50,7 e 49,4\% (Na); 62,0 e $63,4 \%(\mathrm{~K}) ; 73,9$ e 77,3\% (Mg); 64,4 e 68,8\% (S); 76,7 e $82,2 \%(\mathrm{Fe}) ; 68,8$ e $66,9 \%(\mathrm{Zn})$ do total depositado até os 140 dias, nos produtos da gestação.

Em relação à deposição total dos minerais observada durante a gestação, há dificuldade em se comparar os resultados obtidos, em face da inexistência de informações com a espécie caprina. Entretanto, diante dos resultados existentes na literatura para outras espécies, algumas deduções podem ser consideradas. Assim, por exemplo, o conteúdo de cálcio durante a gestação foi de 38,9 e 78,7 $\mathrm{g}$ nas gestações de um e dois fetos, respectivamente, enquanto o ARC (1965) cita conteúdos de 62,3 e 105,6 g para gestações de ovinos produzindo, respectivamente, um cordeiro de $5,9 \mathrm{~kg}$ e dois cordeiros pesando $10,0 \mathrm{~kg}$. Considerando-se que os fetos caprinos pesaram, respectivamente, 3,52 e $6,24 \mathrm{~kg}$ aos 140 dias de gestação, a retenção de Ca obtida para os dois casos seria semelhante.

Utilizando-se esse mesmo raciocínio, Langlands \& Sutherland (1968) obtiveram, em ovinos, uma deposição total de $\mathrm{P}$ de $30,21 \mathrm{~g}$, para gestações com um feto, em que o cordeiro pesou $4,4 \mathrm{~kg}$, enquanto neste trabalho a retenção total foi de $17,8 \mathrm{~g}$ de $\mathrm{P}, \mathrm{o}$ que, proporcionalmente ao peso do feto caprino, estaria próximo ao valor observado por esses autores.

Tabela 3 - Comparação entre a retenção de minerais no útero grávido mais úbere (Y) e a sua estimativa pelas equações de regressão*, em cabras alimentadas ad libitum em diferentes estádios de gestação, com um feto

Table 3 - Comparison between the minerals retention in pregnant uterus plus ubber $(Y)$ and its estimation through the regression equation*, in goats fed ad libitum in different stages of gestation, with one foetus

\begin{tabular}{|c|c|c|c|c|c|c|c|c|c|}
\hline $\begin{array}{l}\text { Tempo } \\
\text { Time }\end{array}$ & $\begin{array}{c}\text { Estimativa } \\
\text { Estimate }\end{array}$ & $\mathrm{Ca}(\mathrm{g})$ & $P(g)$ & $\mathrm{Na}(\mathrm{g})$ & $\mathrm{K}(\mathrm{g})$ & $\mathrm{Mg}(\mathrm{g})$ & $S(g)$ & $\mathrm{Fe}(\mathrm{mg})$ & $\mathrm{Zn}$ (mg) \\
\hline \multirow{4}{*}{50 dias (days) } & $\mathrm{Y}$ & 0,18 & 0,43 & 0,89 & 0,51 & 0,05 & 0,29 & 13,46 & 3,56 \\
\hline & $\mathrm{Y} 1$ & 1,18 & 1,06 & 1,13 & 1,06 & 0,15 & 0,44 & 27,17 & 8,52 \\
\hline & $\mathrm{Y} 2$ & 0,88 & 0,89 & 1,97 & 1,21 & 0,09 & 0,50 & 21,32 & 7,34 \\
\hline & $\mathrm{Y} 3$ & 0,25 & 0,51 & 1,06 & 0,56 & 0,05 & 0,59 & 26,89 & 4,32 \\
\hline \multirow{4}{*}{100 dias (days) } & $\mathrm{Y}$ & 7,43 & 4,89 & 6,50 & 5,06 & 0,55 & 1,95 & 134,29 & 35,13 \\
\hline & $\mathrm{Y} 1$ & 7,04 & 4,60 & 6,41 & 5,51 & 0,82 & 2,21 & 130,52 & 32,63 \\
\hline & $\mathrm{Y} 2$ & 7,21 & 4,71 & 5,71 & 4,61 & 0,53 & 1,90 & 132,99 & 33,50 \\
\hline & $\mathrm{Y} 3$ & 7,43 & 4,89 & 6,46 & 5,05 & 0,55 & 1,82 & 130,69 & 35,01 \\
\hline \multirow{4}{*}{140 dias (days) } & $\mathrm{Y}$ & 38,88 & 17,84 & 13,18 & 13,31 & 2,11 & 5,48 & 575,19 & 112,59 \\
\hline & $\mathrm{Y} 1$ & 38,91 & 17,86 & 13,20 & 13,07 & 1,99 & 5,36 & 575,50 & 112,92 \\
\hline & $\mathrm{Y} 2$ & 38,90 & 17,86 & 13,35 & 13,39 & 2,11 & 5,49 & 575,28 & 112,83 \\
\hline & Y3 & 38,88 & 17,84 & 13,19 & 13,31 & 2,11 & 5,50 & 575,50 & 112,59 \\
\hline
\end{tabular}

\footnotetext{
* $Y 1: \ln Y=A-B e^{-C X}$

$Y 2: Y=A e^{B X}$

$Y 3: \ln Y=A+B X+C X^{2}$
}

R. Bras. Zootec., v.32, n.2, p.425-430, 2003 
Tabela 4 - Comparação entre a retenção de minerais no útero grávido mais úbere (Y) e a sua estimativa pelas equações de regressão*, em cabras alimentadas ad libitum em diferentes estádios de gestação, com dois fetos

Table 4 - Comparison between the minerals retention in pregnant uterus plus ubber $(Y)$ and its estimation through the regression equation ${ }^{*}$, in goats feed ad libitum in different stages of gestation, with two foetus

\begin{tabular}{lccccccrrr}
\hline $\begin{array}{l}\text { Tempo } \\
\text { Time }\end{array}$ & $\begin{array}{c}\text { Estimativa } \\
\text { Estimate }\end{array}$ & $\mathrm{Ca}(\mathrm{g})$ & $\mathrm{P}(\mathrm{g})$ & $\mathrm{Na}(\mathrm{g})$ & $\mathrm{K}(\mathrm{g})$ & $\mathrm{Mg}(\mathrm{g})$ & $\mathrm{S}(\mathrm{g})$ & $\mathrm{Fe}(\mathrm{mg})$ & $\mathrm{Zn}(\mathrm{mg})$ \\
\hline \multirow{4}{*}{50 dias (days) } & $\mathrm{Y}$ & 0,29 & 0,58 & 1,23 & 0,67 & 0,05 & 0,31 & 15,36 & 4,20 \\
& $\mathrm{Y} 1$ & 1,46 & 1,70 & 3,59 & 2,09 & 0,42 & 0,71 & 28,77 & 13,69 \\
& $\mathrm{Y} 2$ & 0,77 & 1,40 & 3,32 & 1,85 & 0,12 & 0,61 & 19,96 & 11,86 \\
& $\mathrm{Y} 3$ & 0,46 & 0,59 & 1,23 & 0,68 & 0,06 & 0,31 & 15,57 & 4,21 \\
& & & & & & & & \\
& $\mathrm{Y}$ & 10,32 & 7,86 & 10,84 & 7,78 & 0,83 & 2,90 & 174,73 & 51,28 \\
100 dias (days) & $\mathrm{Y} 1$ & 9,87 & 7,44 & 9,29 & 7,08 & 1,55 & 2,72 & 171,10 & 50,27 \\
& $\mathrm{Y} 2$ & 10,06 & 7,58 & 9,46 & 7,21 & 0,81 & 2,77 & 173,74 & 51,28 \\
& $\mathrm{Y} 3$ & 10,11 & 7,86 & 10,84 & 7,78 & 0,83 & 2,89 & 174,75 & 54,60 \\
& & & & & & & & \\
140 dias (days) & $\mathrm{Y}$ & 78,75 & 29,15 & 21,44 & 21,27 & 3,65 & 9,29 & 981,10 & 164,75 \\
& $\mathrm{Y} 1$ & 78,73 & 29,20 & 21,83 & 21,40 & 3,15 & 9,31 & 980,79 & 165,47 \\
& $\mathrm{Y} 2$ & 78,75 & 29,19 & 21,84 & 21,40 & 3,65 & 9,31 & 981,12 & 165,44 \\
& $\mathrm{Y} 3$ & 78,70 & 29,14 & 21,44 & 21,27 & 3,65 & 9,28 & 981,00 & 164,74 \\
\hline
\end{tabular}

* $Y 1: \ln Y=A-B e^{-C X}$
$Y 2: Y=A e^{B X}$

$Y 3: \ln Y=A+B X+C X^{2}$

\section{Conclusões}

O modelo exponencial polinomial $(\ln \mathrm{Y}=\mathrm{A}+\mathrm{Bx}$ $+\mathrm{Cx}^{2}$ ) melhor representou o comportamento biológico da retenção de minerais em cabras gestantes.

\section{Literatura Citada}

AGRICULTURAL AND FOOD RESEARCH COUNCIL AFRC. A reapraisal of the calcium and phosphorus requirements of sheep and cattle. Report 6 Nutrition Abstract and Review (Série B), v.61, n.9, p.573-612, 1991.

AGRICULTURAL RESEARCH COUNCIL - ARC. The nutrient requirements of ruminant livestock:technical review. London: Agricultural Research Council Working Party. 1965. 264p.

AGRICULTURAL RESEARCH COUNCIL - ARC. The nutrient requirements of ruminant livestock: technical review. London: Agricultural Research Council Working Party. 1980. 351p.

ANDERSON, R.R.; HARNESS, J.R.; SNEAD, A.F. et al. Mamary growth pattern in goats during pregnancy and lactation. Journal of Dairy Science, v.64, n.3, p.427-32, 1981.

ANDERSON-SPRECHER, R. Model comparisons and R ${ }^{2}$. Am. Statn., v.48, n.2, p.113-7, 1994.

DOTI, J. L.; ADIBI, E.Econometric analysis: an applications approach. New Jersey: Prentice-Hall, 1988. 476p.

FANDINO, B.A.R. Exigências nutricionais de proteína de cabras em gestação. Viçosa, MG: Universidade Federal de Viçosa, 1989. 89p. Dissertação (Mestrado em Zootecnia) Universidade Federal de Viçosa, 1989.

FERREL, C.L.; LASTER, D.B.; PRIOR, R.L. Mineral accretion during prenatal growth of cattle. Journal of Animal Science, v.54, n.3, p.618-24, 1982.

FIELD, A.C.; SUTTLE, N.F. Retention of calcium, phosphorus, magnesium by the developing sheep foetus. Journal of Agriculture Science, v.69, p.417-23, 1967.
GRAHAM, N.M.C. Energy exchanges of pregnant and lactating ewes. Australian Journal of Agriculture Research, v.15, p.127-41, 1964.

KADU, M.; KAIKINI, A. Prenatal development of caprine foetus. Indian Journal of Animal Science, v.57, n.9, p.962-969, 1987.

KOONG, L.J.; GARRETT, W.N.; RATTRAY, P.V. A dynamic description of fetal growth in sheep. Journal of Animal Science, v.41, n.4, p.1065-1068, 1975.

LANGLANDS, J.P.; SUTHERLAND, H.A.M. An estimate of the nutrients utilized for pregnancy by merino sheep.British Journal of Nutrition, v.22, p.217-27, 1968.

LODGE, G.A.; HEANEY, D.P. Composition of weight change in the pregnancy ewe. Canadian Journal of Animal Science, v.53, n.1, p.95-105, 1973.

MOULIN, C.H.S. Exigências energéticas de cabras em gestação. Viçosa, MG: Universidade Federal de Viçosa, 1991, 110p. Dissertação (Mestrado em Zootecnia) - Universidade Federal de Viçosa, 1981.

RATTRAY, P.V.; GARRET, W.N.; EAST, N.E. et al. Efficiency of utilization of metabolizable energy during pregnancy and the energy requirements for pregnancy in sheep. Journal of Animal Science, v.38, n.2, p.383-93, 1974.

ROBINSON, J.J.; BONALD, Mc.; PRASER, I.C. et al. Studies on reproduction in prolific ewes. I. Growth of the products of conception. Journal of Agriculture Science, v.88, p.539-52, 1977.

SILVA, D.J. Análises de alimentos (métodos químicos e biológicos). $1^{\text {a }}$ reimpressão. Viçosa, MG: Universidade Federal de Viçosa, 1998. 166p.

STATISTICAL ANALYSIS SYSTEM - SAS. user's guide: Stat, version 6, 4 ed. Cary, North Caroline: SAS Institute. v.2, 1989. 846p.

WILLET, J.B.; SINGER, J.D. Another cautionary note about $\mathrm{R}^{2}$ : Its use in weighted least-squares regression analysis.Journal of the American Statistical Association, v.42, n.3, p.236-238, 1988.

Recebido em: 11/09/01 Aceito em: 19/08/02 